\title{
On Pointwise Feedback Invariants of Linear Parameter-varying Systems
}

\author{
R. Marta García Fernández ${ }^{*}$, Miguel V. Carriegos \\ Departamento de Matemáticas, Universidad de León, 24071 León, Spain
}

Copyright (C) 2017 by authors, all rights reserved. Authors agree that this article remains permanently open access under the terms of the Creative Commons Attribution License 4.0 International License

\begin{abstract}
Linear systems with constant real coefficients are completely described in terms of feedback actions. In this paper the problem is studied in the framework of linear systems where coefficients depending continuously on a set of parameters. Some invariants are given as well as criteria to find a complete classification in low dimension.
\end{abstract}

Keywords Feedback Classification, Systems over Commutative Rings, Controllability

\section{Introduction}

Let's consider the family of linear control systems

$$
\Sigma(\lambda)=\left\{\begin{array}{l}
\dot{x}=A(\lambda) x(t)+B(\lambda) u(t) \\
y(t)=C(\lambda) x(t)
\end{array}\right.
$$

where $x(t)$ is the $n$-dimensional vector of states, $u(t)$ is the $m$-dimenional vector of external inputs and $y(t)$ is the $p$-dimensional vector of outputs. Matrices $A(\lambda), B(\lambda)$ and $C(\lambda)$ depend continuously on some parameters $\lambda$ living in a compact topological space $\Lambda$. We'll denote by $C(\Lambda, \mathbb{R})$ the ring of continuous real functions defined on $\Lambda$ with pointwise sum and product. This is a commutative ring where $1_{C(\Lambda, \mathbb{R})}$ and $0_{C(\Lambda, \mathbb{R})}$ are respectively the constant functions $\lambda \mapsto 1$ and $\lambda \mapsto 0$.

It is well known that if the matrices have constant coefficients then there is a canonical form for $\Sigma$, the Brunovsky's Canonical Form [5].

Our main goal in this paper is to stablish the so-called pointwise feedback equivalence, see [8], for systems over $C(\Lambda, \mathbb{R})$. This equivalence is just given by all evaluations of parameters of the given linear systems. Therefore pointwise feedback equivalence is studied at every point just like classical feedback equivalence for linear systems with constant coefficients.

We also are interested in stablish global invariants of linear systems for such pointwise feedback equivalence.
Here some sets of zeroes of functions will arise. A complete set of invariants will be given. Despite this classification result, we are not given canonical forms for pointwise feedback action. This is left as a future work.

The paper is organized as follows. Section 2 reviews main results involving feedback equivalence of linear systems which are traslated to the study of pointwise feedback equivalence. Section 3 reviews determinantal ranks of matrices in order to get main pointwise feedback invariants which are introduced in section 4 . This section 4 is also devoted to prove that the set of zeroes of determinantal ideals of reachability maps are invariant for the pointwise feedback equivalence. Low dimensional cases $n \leq 5$ are completely described and characterized in section 5. Finally, we list our conclusions.

\section{Feedback actions and feedback classification}

Let $R$ be a commutative ring with unit $1 \neq 0$. A $m$ input, $n$-dimensional linear system over $R$ is just a pair of matrices $\Sigma=(A, B) \in R^{n \times n} \times R^{n \times m}$ representing the right-hand-side (dynamic) equation

$$
x^{+}(t)=A x(t)+B u(t),
$$

where $x^{+}(t)$ represents time-derivative in continuous time framework or time-shift for discrete systems.

Linear systems $\Sigma^{\prime}=\left(A^{\prime}, B^{\prime}\right)$ and $\Sigma$ are equivalent (feedback) when $\Sigma$ can be transformed to $\Sigma^{\prime}$ by one element of the feedback group $\mathbf{F}_{n m}(R)$ and we will note this by $\Sigma \sim \Sigma$. We recall that feedback group $\mathbf{F}_{n m}(R)$ is the generated group by the following three types of transformations:

(1) $A \longrightarrow A^{\prime}=P A P^{-1}, B \longrightarrow B^{\prime}=P B$ for some invertible matrix $P$. The transformation is a consequence of a change of base in $R^{n}$, the state module.

(2) $A \longrightarrow A, B \longrightarrow B^{\prime}=B Q$ for some invertible matrix $Q$. The transformation is a consequence of change of base in $R^{m}$, the input module. 
(3) $A \longrightarrow A^{\prime}=A+B K, B \longrightarrow B$ for some $m \times n$ matrix $K$ which is called a feedback matrix.

Note 2.1. The feedback classification problem is wild in the sense of Representation Theory (see [4]). Hence it is an open problem in the general case and it is unlikely to be solvable. However in some cases it is possible to find solutions: When $R=\mathbb{K}$ is a field the problem is known as classical case and a classical result of Brunovsky [5, 13, 19] characterizes the class of equivalence of $\Sigma$ by the action of the feedback group, see below.

Throughout this paper we focus on commutative ring $R=C(\Lambda, \mathbb{R})$ of real valued continuous functions defined on compact topological space $\Lambda$. Note that since $\Lambda$ is compact then maximal ideals $\mathfrak{m}$ of $R$ are in one to one correspondence with points in $\Lambda$, that is, given a maximal $\mathfrak{m}$ of $R$ there is a unique point $\lambda_{\mathfrak{m}} \in \Lambda$ such that $f\left(\lambda_{\mathfrak{m}}\right)=0$ for every $f \in \mathfrak{m}$, and conversely, given a point $\lambda \in \Lambda$, the set $\mathfrak{m}_{\lambda}=\{f: f(\lambda)=0\}$ is a maximal of $R$ (the reader can see [1] for details).

Let be $\Sigma=(A, B)$ a linear dynamical system over $R=C(\Lambda, \mathbb{R})$. Then linear system over $\mathbb{R} \cong R / \mathfrak{m}_{\lambda_{0}}$ obtained by extension of scalars $C(\Lambda, \mathbb{R}) \longrightarrow C(\Lambda, \mathbb{R}) / \mathfrak{m}_{\lambda_{0}}$ sending $f \mapsto f\left(\lambda_{0}\right)$ is just obtained by the evaluation at the point $\lambda_{0} \in \Lambda$; that is, $\Sigma\left(\lambda_{0}\right)=\left(A\left(\lambda_{0}\right), B\left(\lambda_{0}\right)\right)$.

Now we recall the definition of pointwise feedback equivalence of linear systems as introduced in [8].

Definition 2.2. Linear systems $\Sigma$ and $\Sigma^{\prime}$ are pointwise feedback equivalent if systems $\Sigma(\lambda)=(A(\lambda), B(\lambda))$ and $\Sigma^{\prime}(\lambda)=\left(A^{\prime}(\lambda), B^{\prime}(\lambda)\right)$ over $\mathbb{R}$ are feedback equivalent for all $\lambda \in \Lambda$.

Pointwise feedback equivalence is weaker than feedback equivalence. That is to say,

Theorem 2.3. If systems $\Sigma=(A, B)$ and $\Sigma^{\prime}=$ $\left(A^{\prime}, B^{\prime}\right)$ are feedback equivalent via the action of element $(P, Q, K) \in \mathbf{F}_{n m}(C(\Lambda, \mathbb{R}))$ then $\Sigma(\lambda)$ and $\Sigma^{\prime}(\lambda)$ are feedback equivalent via $(P(\lambda), Q(\lambda), K(\lambda)) \in \mathbb{F}_{n m}(\mathbb{R})$

Proof. Suppose that $(A, B)$ is feedback equivalent to $\left(A^{\prime}, B^{\prime}\right)$ via $(P, Q, K)$; that is to say

$$
A^{\prime} P=P(A+B K), \text { and } B^{\prime}=P B Q
$$

it follows that above equalities hold on every evaluation; that is

$$
\begin{array}{r}
A^{\prime}(\lambda) P(\lambda)=P(\lambda)(A(\lambda)+B(\lambda) K(\lambda)), \\
B^{\prime}(\lambda)=P(\lambda) B(\lambda) Q(\lambda)
\end{array}
$$

Now the proof is complete once it is assured that $P(\lambda)$ and $Q(\lambda)$ are invertible. But this is trivial because, for instance, $\operatorname{det}(P)$ and $\operatorname{det}(Q)$ are units in $C(\Lambda, \mathbb{R})$ and a fortiori $\operatorname{det}(P(\lambda)) \neq 0$ and $\operatorname{det}(Q(\lambda)) \neq 0$.

We are interested in give a complete (and minimal if possible) set of invariants for pointwise feedback relation. First we recall classical invariants and canonical form for constant linear systems over a field.
Definition 2.4. Let be $\Sigma=(A, B)$ a linear dynamical system of size $(m, n)$ over commutative ring $R$. Consider the $R$-module $N_{i}^{\Sigma}$ generated by columns of the $n \times i m$ matrix $B_{i}^{\Sigma}=\left(B|A B| \ldots \mid A^{i-1} B\right)$. We'll denote by $M_{i}^{\Sigma}$ the quotient module $M_{i}^{\Sigma}=R^{n} / N_{i}^{\Sigma}$.

Above modules are feedback invariant associated to a given linear (over any commutative ring), and they form a minimal complete set in the case of controllable systems over fields. In fact, the following results are well known. First, modules are shown to be feedback invariants:

Theorem 2.5. (cf. [10]) Let be $\Sigma=(A, B)$ a linear dynamical system of size $(m, n)$ over a ring $R$. Then

(i) $(0) \subseteq N_{0}^{\Sigma} \subseteq N_{1}^{\Sigma} \subseteq \ldots \subseteq N_{n}^{\Sigma}$.

(ii) The canonical homomorphism

$$
\begin{gathered}
\varphi_{i}: N_{i}^{\Sigma} / N_{i-1}^{\Sigma} \rightarrow N_{i+1}^{\Sigma} / N_{i}^{\Sigma} \\
\underline{x}+N_{i-1}^{\Sigma} \rightarrow A \underline{x}+N_{i}^{\Sigma}
\end{gathered}
$$

is surjective for $1 \leq i \leq n-1$.

(iii) If $\Sigma$ is feedback equivalent to $\Sigma^{\prime}$ then $N_{i}^{\Sigma}$ and $M_{i}^{\Sigma}$ are isomorphic to $N_{i}^{\Sigma^{\prime}}$ and $M_{i}^{\Sigma^{\prime}}$ respectly, for $1 \leq i \leq n$.

(iv) If $\Sigma$ is a reachable system of simple input $n$ dimensional then the modules $\left\{N_{i}^{\Sigma}\right\}_{1 \leq i \leq n}$ and $\left\{M_{i}^{\Sigma}\right\}_{1 \leq i \leq n}$ are free.

(v) If $\Sigma$ is a Brunovsky system then the modules $\left\{N_{i}^{\Sigma}\right\}_{1 \leq i \leq n}$ and $\left\{M_{i}^{\Sigma}\right\}_{1 \leq i \leq n}$ are free.

Now we deal with the case of fields $\mathbb{K}$ : We prove that above invariant $\mathbb{K}$-vector spaces $N_{i}^{\Sigma}$ form a complete and minimal set of feedback invariants.

Theorem 2.6. (cf. [5]) Let be $\Sigma=(A, B)$ a reachable linear dynamical system of size $(m, n)$ over a field $\mathbb{K}$. Then there exist positive integers $\kappa_{1} \geq \kappa_{2} \geq \cdots \geq \kappa_{s}$ uniquely determined by $\Sigma$ with $n=\kappa_{1}+\kappa_{2}+\cdots+\kappa_{s}$, such that $\Sigma$ is feedback equivalent to the system $\Sigma_{\kappa}=\left(A_{\kappa}, B_{\kappa}\right)$ where $A_{\kappa}$ is the block matrix

$$
A_{\kappa}=\left(\begin{array}{llll}
A_{\kappa_{1}} & 0 & \cdots & 0 \\
0 & A_{\kappa_{2}} & \cdots & 0 \\
\vdots & \vdots & \ddots & \vdots \\
0 & 0 & \cdots & A_{\kappa_{s}}
\end{array}\right)
$$

and block $A_{\kappa_{i}}$ is the $\kappa_{i} \times \kappa_{i}$ matrix

$$
A_{\kappa_{i}}=\left(\begin{array}{cccccc}
0 & 1 & 0 & 0 & \ldots & 0 \\
0 & 0 & 1 & 0 & \ldots & 0 \\
0 & 0 & 0 & 1 & \ldots & 0 \\
\vdots & \vdots & \vdots & \vdots & \ddots & \vdots \\
0 & 0 & 0 & 0 & \ldots & 1 \\
0 & 0 & 0 & 0 & 0 & 0
\end{array}\right)
$$


and

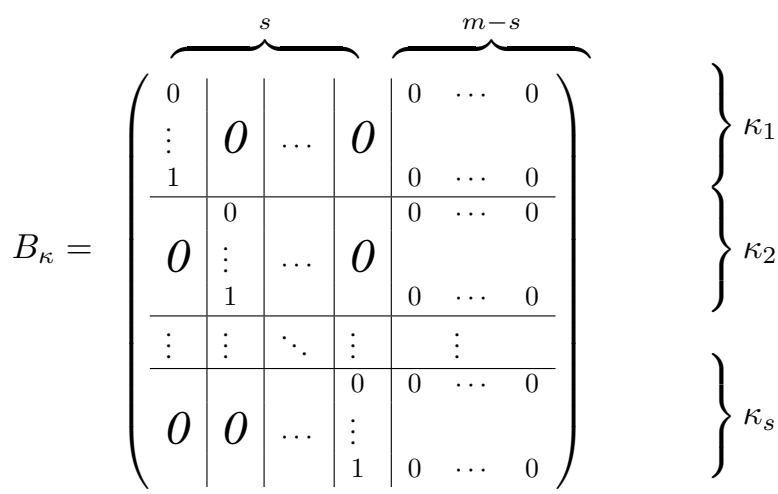

The integers $\kappa=\left\{\kappa_{1}, \kappa_{2}, \ldots, \kappa_{s}\right\}$ are called the Kronecker indices of $\Sigma$. They are a complete set of invariants for $\Sigma$ by the action of the feedback group.

Proof. See [5] or [10].

Above linear system $\Sigma_{\kappa}=\left(A_{\kappa}, B_{\kappa}\right)$ is called Brunovsky's Canonical Form associated to indices $\kappa$.

Note that if $m=1$ and system is controllable it is easy to see that there is just one nonzero index and $\kappa_{1}=n$ hence $s=1$ in above matrices and consequently Brunovsky's Canonical Form in this case is just the Canonical Controller Form

Brunovsky's Canonical form gives rise a complete and minimal set of feedback invariants for controllable linear systems over a field. This is the case of $R=\mathbb{R}$, which is the base for our study. To be precise, the following result summarizes the list of invariants.

Theorem 2.7. (cf.[10]) Let be $\Sigma=(A, B)$ a reachable linear dynamical system of size $(m, n)$ over $\mathbb{R}$. Then the feedback equivalence class of $\Sigma$ is characterized for each one of the following sets:

(i) The Kronecker's indices $\left\{\kappa_{i}\right\}_{1 \leq i \leq s}$

(ii) $\left\{\operatorname{dim}\left(N_{i}^{\Sigma}\right)\right\}_{1 \leq i \leq n}$

(iii) $\left\{\operatorname{dim}\left(N_{i}^{\Sigma} / N_{i-1}^{\Sigma}\right)\right\}_{1 \leq i \leq n}$

Proof. See [10].

According the above result $\left\{\operatorname{rank}_{R} M_{i}^{\Sigma}\right\}_{1<i<n}$ is a complete set of invariants for the class of feedback of a Brunovsky form. If $K$ is a field and $\Sigma=(A, B)$ a reachable dynamical system $\operatorname{rank}\left(M_{i}^{\Sigma}\right)=n-\operatorname{rank}\left(\tilde{B}_{i}^{\Sigma}\right)$ and in consequence $\left\{\operatorname{rank}\left(\tilde{B}_{i}^{\Sigma}\right)\right\}_{1 \leq i \leq n}$ is the list of invariants we need:

Theorem 2.8. A complete set of pointwise feedback invariants for the reachable linear system $\Sigma(\lambda)=(A(\lambda), B(\lambda))$ is given by the one (and hence all) follo-wing data:

(i) $\operatorname{dim}_{\mathbb{R}}\left(N_{i}^{\Sigma(\lambda)}\right), 1 \leq i \leq n, \lambda \in \Lambda$

(ii) $\left\{\operatorname{dim}\left(N_{i}^{\Sigma(\lambda)} / N_{i-1}^{\Sigma(\lambda)}\right)\right\}, 1 \leq i \leq n, \lambda \in \Lambda$ (iii) $\left\{\operatorname{rank}_{\mathbb{R}}\left(\tilde{B}_{i}^{\Sigma(\lambda)}\right)\right\}, 1 \leq i \leq n, \lambda \in \Lambda$

Proof. Two reachable linear systems $\Sigma$ and $\Sigma^{\prime}$ over $C(\Lambda, \mathbb{R})$ are pointwise feedback equivalence if and only if (by definition) linear systems $\Sigma(\lambda)$ and $\Sigma^{\prime}(\lambda)$ are feedback equivalent over $\mathbb{R}$. By Brunovsky's Theorem this is equivalent to $\operatorname{dim}_{\mathbb{R}}\left(N_{i}^{\Sigma(\lambda)}\right)=\operatorname{dim}_{\mathbb{R}}\left(N_{i}^{\Sigma^{\prime}(\lambda)}\right)$, for all $1 \leq i \leq n$, $\lambda \in \Lambda$. Hence data $(i)$ is a complete set of pointwise feedback equivalence. Statements $($ ii $)$ and $($ iii $)$ are proved in the same way.

\section{Determinantal ranks}

Note that pointwise feedback invariants found in above section involves a potentially infinite data (for instance, when compact topological space $\Lambda$ is infinite). Thus we need to refine the result in order to find a minimal complete set of pointwise feedback invariants. This section is devoted to briefly review determinantal rank of a matrix and to compute the determinantal ranks of Brunovsky's canonical forms in order to find our invariants in terms of determinantal ranks in the sequel.

Let be $M=\left(a_{i j}\right)$ an $n \times m$ matrix with entries in $R$ and let be $i$ a nonnegative integer. The $i$-th determinantal ideal of $M$, denoted by $\mathcal{U}_{i}(M)$, is the ideal of $R$ generated by all the $i \times i$ minors of $M$. By construction we have

$$
R=\mathcal{U}_{0}(M) \supseteq \mathcal{U}_{1}(M) \supseteq \ldots \supseteq \mathcal{U}_{i}(M) \supseteq \ldots
$$

and $\mathcal{U}_{i}(M)=0$ for $i>\min \{m, n\}$. The rank of $M$, denoted by $\operatorname{rank}_{R}(M)$, is the largest $i$ such that $\mathcal{U}_{i}(M) \neq 0$. Then $\Sigma$ is reachable if and only if $\mathcal{U}_{n}\left(B_{n}^{\Sigma}\right)=R$.

Now we give a technical result of characterization of Brunovsky's canonical forms $\Sigma_{\kappa}=\left(A_{\kappa}, B_{\kappa}\right)$ depending on the sequence of determinantal ideals. Fist, we give with some definitions and notations.

Definition 3.1. Let be $n \in \mathbb{N}$ and $\kappa=\left\{\kappa_{1}, \kappa_{2}, \ldots, \kappa_{s}\right\}$ with $\kappa_{1} \geq \kappa_{2} \geq \cdots \geq \kappa_{s}$ a partition of $n$. We call dual partition of $\kappa$ to the partition $\eta=\left\{n_{1}, n_{2}, \ldots, n_{p}\right\}$ with $n_{1} \geq n_{2} \geq \cdots \geq n_{p}$ of $n$ where $n_{i}$, is the number of $\kappa_{j}$ which are more or equal than $i$.

Note 3.2. The application $\kappa \rightarrow \eta$ is biyective on the set of partitions of $n$. See [2].

Note 3.3. Note that $n_{1}=s$.

Theorem 3.4. Let be $\kappa=\left\{\kappa_{1}, \kappa_{2}, \ldots, \kappa_{s}\right\}$ a partition of $n$ and $\eta=\left\{n_{1}, n_{2}, \ldots, n_{p}\right\}$ be its associated dual partition. The following conditions are equivalent.

(i) $\kappa$ are the Kronecker indices of system $\Sigma=(A, B)$

(ii) For $1 \leq i \leq p-1$ we have

$$
\begin{aligned}
\mathcal{U}_{n_{1}+n_{2}+\ldots+n_{i}}\left(\tilde{B}_{i}^{\Sigma_{\kappa}}\right) & =\mathbb{K} \\
\mathcal{U}_{n_{1}+n_{2}+\ldots+n_{i}+1}\left(\tilde{B}_{i}^{\Sigma_{\kappa}}\right) & =(0)
\end{aligned}
$$

and for $i=p$

$$
\mathcal{U}_{n_{1}+n_{2}+\ldots+n_{p}}\left(\tilde{B}_{p}^{\Sigma_{\kappa}}\right)=\mathbb{K}
$$


Proof. By Brunovsky Theorem, 2.6,

$$
A=\left(\begin{array}{cccc}
E_{1} & & & \\
& E_{2} & & \\
& & \ddots & \\
& & & E_{s}
\end{array}\right),
$$

where the matrix

$$
E_{i}=\left(\begin{array}{ccccc}
0 & 1 & 0 & \ldots & 0 \\
0 & 0 & 1 & \ddots & 0 \\
\vdots & \vdots & \vdots & \ddots & 0 \\
0 & 0 & 0 & \ldots & 1 \\
0 & 0 & 0 & \ldots & 0
\end{array}\right)
$$

is of dimension $\kappa_{i} \times \kappa_{i}$ and

$$
B=\left(\begin{array}{lllllll}
e_{1} & 0 & \cdots & 0 & 0 & \cdots & 0 \\
0 & e_{2} & \cdots & 0 & 0 & \cdots & 0 \\
\vdots & \vdots & \ddots & \vdots & \vdots & \ddots & \vdots \\
0 & 0 & 0 & e_{s} & 0 & \cdots & 0
\end{array}\right)
$$

where $e_{i}=(0, \ldots, 0,1)^{t}$ is of dimension $\kappa_{i} \times 1$. Then the following properties can be easily verified.

i)

$$
A^{h} B=\left(\begin{array}{lllllll}
E_{1}^{h} e_{1} & 0 & \cdots & 0 & 0 & \cdots & 0 \\
0 & E_{2}^{h} e_{2} & \cdots & 0 & 0 & \cdots & 0 \\
\vdots & \vdots & \ddots & \vdots & \vdots & & \vdots \\
0 & 0 & \cdots & E_{s}^{h} e_{s} & 0 & \cdots & 0
\end{array}\right)
$$

ii) If $h>\kappa_{i}$ is $E_{i}^{h} e_{i}=0$

iii) $\operatorname{Im}\left(A^{h} B\right) \cap \operatorname{Im}\left(A^{h^{\prime}} B\right)=\{0\}$ if $h \neq h^{\prime}$

Let be $n_{1}$ the number of $\kappa_{j}$ greater than or equal to 1 , then the definition $n_{i}$ and by the properties i) and ii) it is followed

$$
\begin{aligned}
& \operatorname{rank}(B)=s=n_{1}, \\
& \operatorname{rank}(A B)=n_{2}, \\
& \operatorname{rank}\left(A^{2} B\right)=n_{3}, \\
& \operatorname{rank}\left(A^{3} B\right)=n_{4}, \\
& \vdots \\
& \operatorname{rank}\left(A^{n_{p}-1} B\right)=n_{p},
\end{aligned}
$$

and by property iii)

$$
\operatorname{rank}\left(\tilde{B}_{i}^{\Sigma_{\kappa}}\right)=n_{1}+n_{2}+\ldots+n_{i}
$$

for $1 \leq i \leq p$, or equivalently

$$
\begin{aligned}
& \mathcal{U}_{n_{1}+n_{2}+\ldots+n_{i}}\left(\tilde{B}_{i}^{\Sigma_{\kappa}}\right)=\mathbb{K} \\
& \mathcal{U}_{n_{1}+n_{2}+\ldots+n_{i}+1}\left(\tilde{B}_{i}^{\Sigma_{\kappa}}\right)=(0)
\end{aligned}
$$

for $1 \leq i \leq p$.
Conversely, by the equalities

$$
\begin{aligned}
& \operatorname{rank}\left(\tilde{B}_{i}^{\Sigma_{\kappa}}\right)=n_{1}+n_{2}+\ldots+n_{i} \\
& \operatorname{rank}\left(\tilde{B}_{i-1}^{\Sigma_{\kappa}}\right)=n_{1}+n_{2}+\ldots+n_{i-1}
\end{aligned}
$$

and the property iii) before it is followed

$$
\operatorname{rank}\left(A^{i-1} B\right)=n_{i}
$$

In consequence,

$$
\begin{aligned}
& \operatorname{rank}(B)=n_{1}=s, \\
& \operatorname{rank}(A B)=n_{2}, \\
& \operatorname{rank}\left(A^{2} B\right)=n_{3}, \\
& \vdots \\
& \operatorname{rank}\left(A^{p-1} B\right)=n_{p},
\end{aligned}
$$

with

$$
n_{1} \geq n_{2} \geq \cdots \geq n_{p}
$$

By the definition of $n_{i}$ (that is

$n_{1}$ is equal to the number of $\kappa_{j}$ greater than or equal to 1 , $n_{2}$ is equal to the number of $\kappa_{j}$ greater than or equal to 2 ,

$n_{p}$ is equal to the number of $\kappa_{j}$ greater than or equal to $\mathrm{p}$ )

it is obtained $\kappa_{i}$ and so the complete set of invariants for the Brunovsky form $\Sigma_{\kappa}$, (for which the partition $\eta=$ $\left\{n_{1}, n_{2}, \ldots, n_{p}\right\}$ is its dual associated partition).

The following properties of Brunovsky canonical forms over any field $\mathbb{K}$ are easily derived:

Corollary 3.5. A Brunovsky canonical form $\Sigma_{\kappa}=$ $\left(A_{\kappa}, B_{\kappa}\right)$ over the field $\mathbb{K}$ verifies

$$
\mathcal{U}_{i}\left(\tilde{B}_{i}^{\Sigma_{\kappa}}\right)=\mathbb{K} \quad 1 \leq i \leq n .
$$

Corollary 3.6. A Brunovsky canonical form $\Sigma_{\kappa}=$ $\left(A_{\kappa}, B_{\kappa}\right)$ over the field $\mathbb{K}$ verifies

$\operatorname{rank}\left(\tilde{B}_{i}^{\Sigma_{\kappa}}\right)=n_{1}+n_{2}+\ldots+n_{i} \geq i \quad$ for $1 \leq i \leq p$ where $\eta=\left\{n_{1}, \ldots, n_{p}\right\}$ is the dual partition of $\kappa$. In particular

$$
\operatorname{rank}\left(\tilde{B}_{p}^{\Sigma_{\kappa}}\right) .=n_{1}+n_{2}+\ldots+n_{p}=n .
$$

Corollary 3.7. The following conditions hold for $\mathbb{K}$-vector spaces $N_{i}^{\Sigma_{\kappa}}$ generated by columns of the $n \times i m$ matrix.

$$
\tilde{B}_{i}^{\Sigma_{\kappa}}=\left(B_{\kappa}\left|A_{\kappa} B_{\kappa}\right| \ldots \mid A_{\kappa}^{i-1} B_{\kappa}\right)
$$

associated to Brunovsky canonical form $\Sigma_{\kappa}$

(i) $\operatorname{dim}\left(N_{i}^{\Sigma_{\kappa}}\right) \leq \operatorname{dim}\left(N_{i+1}^{\Sigma_{\kappa}}\right)$ for all $i=1,2, \ldots$

(ii) If $\operatorname{dim}\left(N_{t}^{\Sigma_{\kappa}}\right)=\operatorname{dim}\left(N_{t+1}^{\Sigma_{\kappa}}\right)$ then

$$
\operatorname{dim}\left(N_{t}^{\Sigma_{\kappa}}\right)=\cdots=\operatorname{dim}\left(N_{n}^{\Sigma_{\kappa}}\right)=n .
$$


Now, we are ready to give a characterization of pointwise feedback equivalence in terms of dimensions of pointwise invariants.

Theorem 3.8. Let be $\Sigma=(A, B)$ and $\Sigma^{\prime}=\left(A^{\prime}, B^{\prime}\right)$ two reachable linear dynamical systems of size $(m, n)$ over $R=C(\Lambda, \mathbb{R})$. For $\lambda_{0} \in \Lambda$, the following conditions are equivalent.

(i) $\Sigma\left(\lambda_{0}\right) \sim \Sigma_{\kappa}$ where $\Sigma_{\kappa}=\left(A_{\kappa}, B_{\kappa}\right)$ is the Brunovsky's linear form associated to the Kronecker's indices $\kappa=\left\{\kappa_{1}, \kappa_{2}, \ldots, \kappa_{s}\right\}$

(ii) Let be $\eta=\left\{n_{1}, n_{2}, \ldots, n_{p}\right\}$ the dual partition of $\kappa$. Then

$$
\operatorname{dim}_{\mathbb{R}}\left(N_{i}^{\Sigma\left(\lambda_{0}\right)}\right)=n_{1}+n_{2}+\ldots+n_{i} ; \quad 1 \leq i \leq p
$$

Proof. (i) $\Rightarrow$ (ii) As $\left(A\left(\lambda_{0}\right), B\left(\lambda_{0}\right)\right) \sim\left(A_{\kappa}, B_{\kappa}\right)$ and $\Sigma_{\kappa}=\left(A_{\kappa}, B_{\kappa}\right)$ is the Brunovsky form over the field $K=$ $\mathbb{R}$, whose associated partition is $\kappa=\left\{\kappa_{1}, \kappa_{2}, \ldots, \kappa_{s}\right\}$, by Theorem 3.4 it is

$$
\left\{\begin{array}{l}
\mathcal{U}_{n_{1}+n_{2}+\ldots+n_{i}}\left(\tilde{B}_{i}^{\Sigma_{\kappa}}\right)=\mathbb{R} \\
\mathcal{U}_{n_{1}+n_{2}+\ldots+n_{i}+1}\left(\tilde{B}_{i}^{\Sigma_{\kappa}}\right)=(0) \quad \text { for } \quad 1 \leq i \leq p
\end{array}\right.
$$

or equivalently

$$
\left\{\begin{array}{l}
\mathcal{U}_{n_{1}+n_{2}+\ldots+n_{i}}\left(\tilde{B}_{i}^{\Sigma\left(\lambda_{0}\right)}\right)=\mathbb{R} \\
\mathcal{U}_{n_{1}+n_{2}+\ldots+n_{i}+1}\left(\tilde{B}_{i}^{\Sigma\left(\lambda_{0}\right)}\right)=(0) \quad \text { for } \quad 1 \leq i \leq p,
\end{array}\right.
$$

then

$$
\operatorname{dim}\left(N_{i}^{\Sigma\left(\lambda_{0}\right)}\right)=\operatorname{rank}\left(\tilde{B}_{i}^{\Sigma\left(\lambda_{0}\right)}\right)=n_{1}+n_{2}+\ldots+n_{i}
$$

for $1 \leq i \leq p$.

(ii) $\Rightarrow$ (i) Conversely, let be $\lambda_{0} \in \Lambda$ such that

$\operatorname{dim}\left(N_{i}^{\Sigma\left(\lambda_{0}\right)}\right)=n_{1}+n_{2}+\ldots+n_{i} \quad$ for $\quad 1 \leq i \leq p$

then

$$
\operatorname{rank}\left(\tilde{B}_{i}^{\Sigma\left(\lambda_{0}\right)}\right)=n_{1}+n_{2}+\ldots+n_{i}=\operatorname{rank}\left(\tilde{B}_{i}^{\Sigma_{\kappa}}\right)
$$

for $1 \leq i \leq p$ Also

$\mathcal{U}_{j}\left(\tilde{B}_{i}^{\Sigma\left(\lambda_{0}\right)}\right)=\mathcal{U}_{j}\left(\tilde{B}_{i}^{\Sigma_{\kappa}}\right)$ for $\quad 1 \leq i \leq n, 1 \leq j \leq n$, and therefore $\Sigma\left(\lambda_{0}\right)$ is feedback equivalent to $\Sigma_{\kappa}$.

\section{The pointwise feedback relation}

Note that if $A=\left(f_{i j}\right)$ is a matrix over $R=C(\Lambda, \mathbb{R})$ then $A(\lambda)$ is the matrix $\left(f_{i j}(\lambda)\right)$. Let be $\Sigma=(A, B)$ and $\Sigma^{\prime}=\left(A^{\prime}, B^{\prime}\right)$ two systems $n$-dimensional with $m$ inputs over $R=C(\Lambda, \mathbb{R})$, if $\Sigma$ is equivalent feedback to $\Sigma^{\prime}$, then $\Sigma(\lambda)$ is equivalent feedback to $\Sigma^{\prime}(\lambda)$ for all $\lambda$. The converse is not true in general, and this motivates the study of following relationship. We say $\Sigma$ and $\Sigma^{\prime}$ are pointwise feedback equivalent if the systems $\Sigma(\lambda)=(A(\lambda), B(\lambda))$ and $\Sigma^{\prime}(\lambda)=\left(A^{\prime}(\lambda), B^{\prime}(\lambda)\right)$ over $\mathbb{R}$ are feedback equivalents for all $\lambda \in \Lambda$. Since reachability is a property that it is preserved by feedback, let us see this concept in the ring $R=C(\Lambda, \mathbb{R})$.

Theorem 4.1. Let be $\Lambda$ a compact topological space and $\Sigma=(A, B)$ be a reachable linear dynamical system of size $(m, n)$ over $R=C(\Lambda, \mathbb{R})$. Then the following conditions are equivalent.

(i) $\Sigma$ is reachable over $C(\Lambda, \mathbb{R})$

(ii) $\Sigma(\lambda)$ is reachable over $\mathbb{R}$, for all $\lambda \in \Lambda$.

Proof. (i) $\Rightarrow$ (ii) If $\Sigma$ is reachable in $C(\Lambda, \mathbb{R})$ then

$$
\mathcal{U}_{n}\left(\tilde{B}^{\Sigma}\right)=\left(f_{1}, f_{2}, \ldots, f_{k}\right)=(1)=C(\Lambda, \mathbb{R})
$$

where $f_{1}, f_{2}, \ldots, f_{k}$ are the minors of order $n$ of the matrix $\tilde{B}^{\Sigma}$.

Since $\tilde{B}^{\Sigma}(\lambda)=\tilde{B}^{\Sigma(\lambda)}$, it follows

$\mathcal{U}_{n}\left(\tilde{B}^{\Sigma(\lambda)}\right)=\mathcal{U}_{n}\left(\tilde{B}^{\Sigma}\right) \otimes C(\Lambda, \mathbb{R}) / \mathfrak{m}_{\lambda}=C(\Lambda, \mathbb{R}) / \mathfrak{m}_{\lambda}$ and in consequence $\operatorname{rank}\left(\tilde{B}^{\Sigma(\lambda)}\right)=n$, or equivalently $\Sigma(\lambda)$ is reachable for all $\lambda$ in $\Lambda$.

(ii) $\Rightarrow$ (i) Conversely, assume

$\mathcal{U}_{n}\left(\tilde{B}^{\Sigma}\right)=\mathcal{U}_{n}\left(B|A B| \ldots \mid A^{n-1} B\right)=\left(f_{1}, f_{2}, \ldots, f_{k}\right)$,

where $f_{1}, f_{2}, \ldots, f_{k}$ are all minors of order $n$ of the matrix $\tilde{B}^{\Sigma}$. As $\Sigma(\lambda)=(A(\lambda), B(\lambda))$ is reachable for all $\lambda$ in $\Lambda$, we have $\operatorname{rank}\left(\tilde{B}^{\Sigma(\lambda)}\right)=n$.

Then the set of zeroes (see below) $Z\left(f_{1}, \ldots, f_{k}\right)=\emptyset$ is empty. Thus ideal generated by $\left\{f_{1}, f_{2}, \ldots, f_{k}\right\}$ is the whole ring. A fortiori $f_{1}^{2}+f_{2}^{2}+\ldots+f_{k}^{2}$ is an unit in $C(\Lambda, \mathbb{R})$. Then $\mathcal{U}_{n}\left(\tilde{B}^{\Sigma}\right)=R$ and $\Sigma$ is a reachable system.

In order to introduce the sets of invariants for pointwise feedback relationship we need to remark usual notation of ideal of zeroes of a function. Let be $\mathfrak{a}$ an ideal of $R=$ $C(\Lambda, \mathbb{R})$. We'll denote by $Z(\mathfrak{a})$ the set

$$
Z(\mathfrak{a})=\{\lambda \in \Lambda / f(\lambda)=0 \quad \text { for all } f \in \mathfrak{a}\}
$$

The following result (see [8]) gives a set of invariants for the pointwise feedback relation.

Theorem 4.2. Let be $\Sigma=(A, B)$ and $\Sigma^{\prime}=\left(A^{\prime}, B^{\prime}\right)$ two reachable linear systems of type $(n, m)$ over the ring $R=$ $C(\Lambda, \mathbb{R})$ then the following conditions are equivalent. 
(i) Systems $\Sigma$ and $\Sigma^{\prime}$ are pointwise feedback equivalents; that is to say, $\Sigma(\lambda)$ and $\Sigma^{\prime}(\lambda)$ are feedback equivalents for $\lambda \in \Lambda$

(ii) For all $1 \leq i, j \leq n$ one has $Z\left(\mathcal{U}_{j}\left(\tilde{B}_{i}^{\Sigma}\right)\right)=$ $Z\left(\mathcal{U}_{j}\left(\tilde{B}_{i}^{\Sigma^{\prime}}\right)\right) \quad 1 \leq i \leq n, \quad 1 \leq j \leq n$

Proof. $(i) \Rightarrow(i i)$. If $\lambda \in Z\left(\mathcal{U}_{j}\left(\tilde{B}_{i}^{\Sigma}\right)\right)$ then $\mathcal{U}_{j}\left(\tilde{B}_{i}^{\Sigma(\lambda)}\right)=(0)$ and hence $\operatorname{dim}\left(N_{i}^{\Sigma(\lambda)}\right)<j$. Since $\Sigma(\lambda)$ and $\Sigma^{\prime}(\lambda)$ are feedback equivalent over $\mathbb{R}$ it follows that it is also satisfied $\operatorname{dim}\left(N_{i}^{\Sigma^{\prime}(\lambda)}\right)<j$ which yields $\lambda \in Z\left(\mathcal{U}_{j}\left(\tilde{B}_{i}^{\Sigma^{\prime}}\right)\right)$. The inverse contention is also proved because feedback equivalence is a equivalence relation, and symmetric property solves the case.

$(i) \Leftarrow(i i)$ Let be $\lambda \in \Lambda$. Observe that $(i i)$ yields that

$$
\begin{aligned}
& \operatorname{dim}\left(N_{i}^{\Sigma(\lambda)}\right)=\min \left\{j: \lambda \in Z\left(\mathcal{U}_{j}\left(\tilde{B}_{i}^{\Sigma}\right)\right)\right\}= \\
& =\min \left\{j: \lambda \in Z\left(\mathcal{U}_{j}\left(\tilde{B}_{i}^{\Sigma^{\prime}}\right)\right)\right\}=\operatorname{dim}\left(N_{i}^{\Sigma(\lambda)}\right)
\end{aligned}
$$

Therefore $\mathbb{R}$ vector spaces $N_{i}^{\Sigma(\lambda)}$ and $N_{i}^{\Sigma^{\prime}(\lambda)}$ are isomorphic for all $i$ and all $\lambda \in \Lambda$ and consequently $\Sigma(\lambda)$ and $\Sigma^{\prime}(\lambda)$ are feedback equivalent for all $\lambda \in \Lambda$ or equivalently systems $\Sigma$ and $\Sigma^{\prime}$ are point wise feedback equivalents.

At this point let us see how the generation of these zeroes sets can be done by only one element.

Theorem 4.3. Sets of zeroes of finitely generated ideals of $C(\Lambda, \mathbb{R})$ can be obtained as the set of zeroes of a single function. That is to say, one has the following properties:

(i) Let be a finitely generated ideal of $C(\Lambda, \mathbb{R})$. Then there exists $a \in \mathfrak{a}$ such that $Z(\mathfrak{a})=Z(a)$

(ii) If $\mathfrak{a} \subseteq \mathfrak{b}$ are finitely generated ideals of $C(\Lambda, \mathbb{R})$ then we can choose $a \in \mathfrak{a}$ and $b \in \mathfrak{b}$ with $a=\lambda b$ such that

$$
Z(\mathfrak{a})=Z(a) \supseteq Z(b)=Z(\mathfrak{b})
$$

Proof. (i) If $\mathfrak{a}$ is generated by $f_{1}, f_{2}, \ldots, f_{k}$ with $f_{i} \in$ $C(\Lambda, \mathbb{R})$, it is enough to consider

$$
a=f_{1}^{2}+f_{2}^{2}+\ldots+f_{k}^{2}
$$

(ii) Let us consider the elements $a^{\prime} \in \mathfrak{a}$ and $b^{\prime} \in \mathfrak{b}$ (for example, built as in the previous item) such that

$$
Z(\mathfrak{a})=Z\left(a^{\prime}\right) \supseteq Z\left(b^{\prime}\right)=Z(\mathfrak{b}) .
$$

The result is followed considered the elements $b=b^{\prime} \in \mathfrak{b}$ y $a=a^{\prime} b^{\prime} \in \mathfrak{a}$. Hence

$Z(a)=Z\left(a^{\prime} b^{\prime}\right)=Z\left(a^{\prime}\right) \cup Z\left(b^{\prime}\right)=Z(\mathfrak{a}) \cup Z(\mathfrak{b})=Z(\mathfrak{a})$.

Theorem 4.4. Let be $\mathfrak{a}$ an ideal of $C(\Lambda, \mathbb{R})$ generated by $f_{1}, f_{2}, \ldots, f_{k}$. The following conditions are equivalent. (i) $\mathfrak{a} \neq C(\Lambda, \mathbb{R})$

(ii) $Z\left(f_{1}\right) \cap Z\left(f_{2}\right) \cap \ldots \cap Z\left(f_{k}\right) \neq \emptyset$

(iii) $f_{1}^{2}+f_{2}^{2}+\ldots+f_{k}^{2}$ is not an unit of $C(\Lambda, \mathbb{R})$

Proof. It is sufficient to note that

$$
\begin{aligned}
& Z(\mathfrak{a})=Z\left(f_{1}^{2}+f_{2}^{2}+\ldots+f_{k}^{2}\right)= \\
& Z\left(f_{1}\right) \cap Z\left(f_{2}\right) \cap \ldots \cap Z\left(f_{k}\right) \neq \emptyset .
\end{aligned}
$$

Recall that Theorem 4.2 states that the $n^{2}$ sets given by

$$
\left\{Z\left(\mathcal{U}_{j}\left(\tilde{B}_{i}^{\Sigma}\right)\right)\right\}
$$

for $1 \leq i \leq n, 1 \leq j \leq n$, are a complete system invariants for pointwise feedback relation. We conclude this section by studying that set of invariants. Main properties are given in the next results.

First of all, note that a reachable linear system over $R$ verifies: $\mathcal{U}_{j}\left(\tilde{B}_{i}^{\Sigma}\right)=R$, for all $j \leq i$. In terms of sets of zeroes of reachability maps, one has the following result:

Theorem 4.5. Let be $\Sigma=(A, B)$ a reachable linear $d y$ namical system of type $(n, m)$ over $R=C(\Lambda, \mathbb{R})$ then one has $Z\left(\mathcal{U}_{j}\left(\tilde{B}_{i}^{\Sigma}\right)\right)=\emptyset$ for all $j \leq i$.

Proof. First note that since system is reachable then $\mathcal{U}_{j}\left(\tilde{B}_{i}^{\Sigma}\right)=R$.

Now, by contradiction, suppose that $\lambda \in Z\left(\mathcal{U}\left(\tilde{B}_{i}^{\Sigma}\right)\right.$ it follows that $f(\lambda)=0$ for all $f \in \mathcal{U}\left(\tilde{B}_{i}^{\Sigma}\right)$ and therefore $1 \notin \mathcal{U}\left(\tilde{B}_{i}^{\Sigma}\right)$, which is a contradiction.

Lemma 4.6. Let be $\Sigma_{\kappa}=\left(A_{\kappa}, B_{\kappa}\right)$ a Brunovsky form of type $(n, m)$ over the field $K$. If $\operatorname{rank}\left(\tilde{B}_{i+1}^{\Sigma_{\kappa}}\right)<j$, then

$$
\operatorname{rank}\left(\tilde{B}_{i}^{\Sigma_{\kappa}}\right)<j-1
$$

Proof. With the notation of Theorem 3.4 we have

$$
\operatorname{rank}\left(\tilde{B}_{i+1}^{\Sigma_{\kappa}}\right)=n_{1}+n_{2}+\ldots+n_{i}+n_{i+1}<j
$$

and as $n_{i+1} \geq 1$, it is followed that

$$
\begin{gathered}
\operatorname{rank}\left(\tilde{B}_{i}^{\Sigma_{\kappa}}\right)=n_{1}+n_{2}+\ldots+n_{i}= \\
\operatorname{rank}\left(\tilde{B}_{i+1}^{\Sigma_{\kappa}}\right)-n_{i+1}<j-n_{i+1} \leq j-1
\end{gathered}
$$

Theorem 4.7. Let be $\Sigma=(A, B)$ a reachable linear $d y$ namical system of type $(n, m)$ with coefficients in the ring $R=C(\Lambda, \mathbb{R})$ then

$$
Z\left(\mathcal{U}_{j}\left(\tilde{B}_{i}^{\Sigma}\right)\right) \supseteq Z\left(\mathcal{U}_{j+1}\left(\tilde{B}_{i+1}^{\Sigma}\right)\right)
$$


Proof. It is immediate from the previous Lemma.

Theorem 4.8. Let be $\Sigma=(A, B)$ a reachable dynamical system of type $(n, m)$ over $R=C(\Lambda, \mathbb{R})$, then

$$
Z\left(\mathcal{U}_{j}\left(\tilde{B}_{i}^{\Sigma}\right)\right)=Z\left(\mathcal{U}_{j+1}\left(\tilde{B}_{i+1}^{\Sigma}\right)\right) \text { for } j \leq 2 i
$$

where

$$
\tilde{B}_{i}^{\Sigma}=\left(B|A B| \ldots \mid A^{i-1} B\right)
$$

Proof. By means of Theorem 4.7 it is enough to prove

$$
Z\left(\mathcal{U}_{j}\left(\tilde{B}_{i}^{\Sigma}\right)\right) \subseteq Z\left(\mathcal{U}_{j+1}\left(\tilde{B}_{i+1}^{\Sigma}\right)\right)
$$

Let be $\lambda_{0} \in Z\left(\mathcal{U}_{j}\left(B|A B| \ldots \mid A^{i-1} B\right)\right)$ and we consider the system $\Sigma\left(\lambda_{0}\right)$, reachable over $\mathbb{R}$, then $\Sigma\left(\lambda_{0}\right)$ is feedback equivalent to a Brunovsky form $\Sigma_{\kappa}=\left(A_{\kappa}, B_{\kappa}\right)$ where $\kappa=\left\{\kappa_{1}, \kappa_{2}, \ldots, \kappa_{s}\right\}$ is a partition of $n$ with $\kappa_{1} \geq$ $\kappa_{2} \geq \cdots \geq \kappa_{s}$.

As

$$
\operatorname{rank}\left(B\left(\lambda_{0}\right)\left|A\left(\lambda_{0}\right) B\left(\lambda_{0}\right)\right| \ldots \mid A^{i-1}\left(\lambda_{0}\right) B\left(\lambda_{0}\right)\right)<j
$$

it is

$$
\operatorname{rank}\left(B_{\kappa}\left|A_{\kappa} B_{\kappa}\right| \ldots \mid A_{\kappa}^{i-1} B_{\kappa}\right)<j,
$$

or equivalent

$$
\operatorname{rank}\left(B_{\kappa}\left|A_{\kappa} B_{\kappa}\right| \ldots \mid A_{\kappa}^{i-1} B_{\kappa}\right) \leq j-1 .
$$

Let us see

$$
\operatorname{rank}\left(B_{\kappa}\left|A_{\kappa} B_{\kappa}\right| \ldots \mid A_{\kappa}^{i} B_{\kappa}\right) \leq j
$$

Arguing by contradiction, assume

$$
\operatorname{rank}\left(B_{\kappa}\left|A_{\kappa} B_{\kappa}\right| \ldots \mid A_{\kappa}^{i} B_{\kappa}\right)>j
$$

we'll have

$$
\operatorname{rank}\left(A_{\kappa}^{i} B_{\kappa}\right)>1,
$$

then $E_{1}^{i} e_{1} \neq 0$ and $E_{2}^{i} e_{2} \neq 0$ and it must be

$$
i<\kappa_{1} \quad \text { and } \quad i<\kappa_{2} \text {. }
$$

But this is the same that

$$
i<\kappa_{2} \leq \kappa_{1},
$$

and taking into account only the column system vectors linearly independent

$$
\left\{e_{1}, e_{2}, A_{\kappa} e_{1}, A_{\kappa} e_{2}, \ldots, A_{\kappa}^{i-1} e_{1}, A_{\kappa}^{i-1} e_{2}\right\}
$$

in the matrix $\tilde{B}_{i}^{\Sigma}=\left(B_{\kappa}\left|A_{\kappa} B_{\kappa}\right| \ldots \mid A_{\kappa}^{i-1} B_{\kappa}\right)$, we have

$$
\operatorname{rank}\left(B_{\kappa}\left|A_{\kappa} B_{\kappa}\right| \ldots \mid A_{\kappa}^{i-1} B_{\kappa}\right) \geq 2 i
$$

but, by hypothesis

$$
\operatorname{rank}\left(B_{\kappa}\left|A_{\kappa} B_{\kappa}\right| \ldots \mid A_{\kappa}^{i-1} B_{\kappa}\right) \leq j-1,
$$

then

$$
j-1 \geq 2 i,
$$

so that

$$
j \geq 2 i+1,
$$

and it is contrary to the course. Therefore, it should be

$$
\operatorname{rank}\left(B_{\kappa}\left|A_{\kappa} B_{\kappa}\right| \ldots \mid A_{\kappa}^{i} B_{\kappa}\right) \leq j
$$

and also

$$
\operatorname{rank}\left(B\left(\lambda_{0}\right)\left|A\left(\lambda_{0}\right) B\left(\lambda_{0}\right)\right| \ldots \mid A^{i}\left(\lambda_{0}\right) B\left(\lambda_{0}\right)\right) \leq j,
$$

then

$$
\lambda_{0} \in Z\left(\mathcal{U}_{j+1}\left(B|A B| \ldots \mid A^{i-1} B\right)\right)
$$

In the table at the Note 4.10 , Theorem 4.7 test contentions chains parallel to the main diagonal, while Theorem 4.8 proves the equalities. In addition, these equalities can not be extended to $j>2 i$, as it is shown in the following result.

Theorem 4.9. Let be $\Sigma=(A, B)$ a reachable linear $d y$ namical system of type $(n, m)$ over $R=C(\Lambda, \mathbb{R})$, and let be $\lambda_{0} \in \Lambda$ such that $\Sigma\left(\lambda_{0}\right) \sim \Sigma_{\kappa}$ where $\kappa=$ $\left\{\kappa_{1}, \kappa_{2}, \ldots, \kappa_{s}\right\}$, with $\eta=\left\{n_{1}, n_{2}, \ldots, n_{p}\right\}$ the dual partition associated checking

$$
\begin{aligned}
2 i & \leq n_{1}+n_{2}+\ldots+n_{i}<j \\
<j+1 & \leq n_{1}+n_{2}+\ldots+n_{i}+n_{i+1},
\end{aligned}
$$

then

$$
\lambda_{0} \in Z\left(\mathcal{U}_{j}\left(\tilde{B}_{i}^{\Sigma}\right)\right) \backslash Z\left(\mathcal{U}_{j+1}\left(\tilde{B}_{i+1}^{\Sigma}\right)\right)
$$

Proof. By Theorem 3.4, and being

$$
n_{1}+n_{2}+\ldots+n_{i}<j
$$

we have

$$
\mathcal{U}_{j}\left(\tilde{B}_{i}^{\Sigma\left(\lambda_{0}\right)}\right)=\mathcal{U}_{j}\left(\tilde{B}_{i}^{\Sigma_{\kappa}}\right)=0
$$

then $\lambda_{0} \in Z\left(\mathcal{U}_{j}\left(\tilde{B}_{i}^{\Sigma}\right)\right)$.

Also, as

$$
j+1 \leq n_{1}+n_{2}+\ldots+n_{i}+n_{i+1}
$$

it is

$$
\mathcal{U}_{j+1}\left(\tilde{B}_{i+1}^{\Sigma\left(\lambda_{0}\right)}\right)=\mathcal{U}_{j+1}\left(\tilde{B}_{i+1}^{\Sigma_{\kappa}}\right) \neq 0
$$

and $\lambda_{0} \notin Z\left(\mathcal{U}_{j+1}\left(\tilde{B}_{i+1}^{\Sigma}\right)\right)$.

Note 4.10. From Theorems 4.5, 4.7 and 4.8 contentions and equalities are deduced between sets $\left\{Z\left(\mathcal{U}_{j}\left(B|A B| \ldots \mid A^{i-1} B\right)\right)\right\}$ for $1 \leq i \leq n$, $1 \leq j \leq n$, that are showed in the following scheme in the Figure 1. 


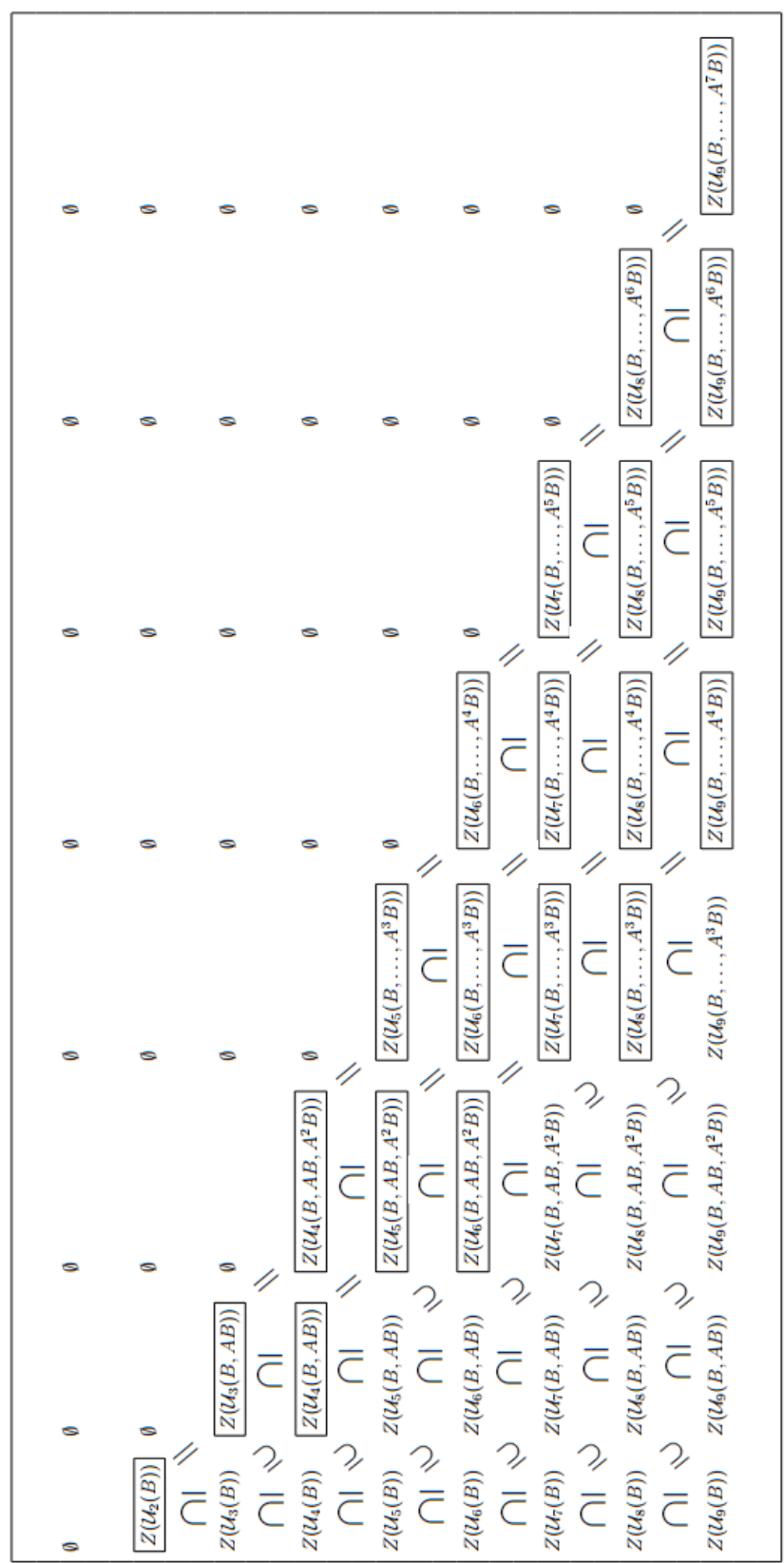

Figure 1. Some relations in $\left\{z\left(\mathcal{U}_{j}\left(B|A B| \ldots \mid A^{i-1} B\right)\right)\right\}$

\section{Low dimensional cases $(n \leq 5)$}

For the cases $n=2, n=3$ and $n=4$ it follows

Corollary 5.1. The sets of zeroes

$$
Z\left(\mathcal{U}_{2}(B)\right)
$$

are a complete set of invariants for the feedback pointwise class of the system $\Sigma=(A, B)$ reachable of type $(2, m)$, over the ring $R=C(\Lambda, \mathbb{R})$.

For systems of type $(3, m)$ we can give the similar result.

Corollary 5.2. The sets of zeroes

$$
Z\left(\mathcal{U}_{2}(B)\right) \supseteq Z\left(\mathcal{U}_{3}(B)\right)
$$

are a complete set of invariants for the feedback pointwise class of the system $\Sigma=(A, B)$ reachable of type $(3, m)$, over the ring $R=C(\Lambda, \mathbb{R})$.

Corollary 5.3. The sets of zeroes

$$
Z\left(\mathcal{U}_{4}(B)\right) \supseteq Z\left(\mathcal{U}_{3}(B)\right) \supseteq Z\left(\mathcal{U}_{4}(B \mid A B)\right) \supseteq Z\left(\mathcal{U}_{2}(B)\right)
$$

are a complete set of invariants for the feedback pointwise class of the system $\Sigma=(A, B)$ reachable of type $(4, m)$, over the ring $R=C(\Lambda, \mathbb{R})$.

After these general reductions, there are another reductions depending on $n$. For example in the case $n=5$ we can give the following result.

Theorem 5.4. Let be $\Sigma=(A, B)$ a reachable linear dynamical system of type $(5, m)$ over the ring $R=C(\Lambda, \mathbb{R})$. Then

$$
\begin{aligned}
& Z\left(\mathcal{U}_{5}(B)\right) \supseteq Z\left(\mathcal{U}_{4}(B)\right) \supseteq Z\left(\mathcal{U}_{5}(B \mid A B)\right) \supseteq \\
& Z\left(\mathcal{U}_{3}(B)\right) \supseteq Z\left(\mathcal{U}_{4}(B \mid A B)\right) \supseteq Z\left(\mathcal{U}_{2}(B)\right)
\end{aligned}
$$

Proof. It is enough to prove

$$
Z\left(\mathcal{U}_{5}\left(B|A B \ldots| A^{n-1} B\right)\right) \supseteq Z\left(\mathcal{U}_{3}(B)\right)
$$

Let be $\lambda_{0} \in Z\left(\mathcal{U}_{3}(B)\right)$ then $\Sigma\left(\lambda_{0}\right)=\left(A\left(\lambda_{0}\right), B\left(\lambda_{0}\right)\right)$ is a reachable system over the field $K=\mathbb{R}$. By Brunovsky theorem, $\Sigma\left(\lambda_{0}\right)$ only can be equivalent feedback to a Brunovsky form $\Sigma_{\kappa}=\left(A_{\kappa}, B_{\kappa}\right)$, where $\kappa$ is one of the following partitions for $n=5$ :

$$
\begin{aligned}
& \kappa_{1}=3 \geq \kappa_{2}=2>0 \quad \text { or } \\
& \kappa_{1}=4 \geq \kappa_{2}=1>0 \quad \text { or } \\
& \kappa_{1}=5>0
\end{aligned}
$$

where $\mathcal{U}_{5}\left(B_{\kappa}, A_{\kappa} B_{\kappa}\right)=(0)$. By the feedback equivalence is

$$
\mathcal{U}_{5}\left(B\left(\lambda_{0}\right), A\left(\lambda_{0}\right) B\left(\lambda_{0}\right)\right)=\mathcal{U}_{5}\left(B_{\kappa}, A_{\kappa} B_{\kappa}\right)=(0),
$$

and $\lambda_{0} \in Z\left(\mathcal{U}_{5}(B \mid A B)\right)$.

\section{Conclusion}

The problem of obtaining invariants in the pointwise feedback equivalence over $R=C(\Lambda, \mathbb{R})$ has been considered.The next step will be to construct a canonical form for each $n$ and the opportunity of stratify the space $\Lambda$ sorting these invariants as a lattice. For example given the table of the Figure 1, open question is to generalize reducing results as Theorem 5.4 and can extend results to the ring $C^{k}(\Lambda, \mathbb{R})$ where $\Lambda$ is a differentiable manifold or to extend results to the ring of holomorphic functions $H(\Omega)$ where $\Omega \subseteq \mathbb{C}$. 


\section{Acknowledgements}

The Instituto Nacional de Ciberseguridad (Spanish National Institute for Cybersecurity) (INCIBE) has partially supported this work.

We are also grateful to the annonymous referee for valuable comments.

\section{REFERENCES}

[1] Atiyah, M.F., Macdonald, I.G. Introduction to Commutative Algebra. Addison-Wesley. (1969).

[2] N.L. Biggs, Discrete Mathematics.(Chap. 26), Oxford University Press, (2003).

[3] J.W. Brewer, J.W. Bunce and F.S. Van Vleck, Linear Systems over Commutative Rings, Marcel Dekker, New York, (1986).

[4] J. Brewer, L. Klingler, On feedback invariants for linear dynamical systems, Linear Algbera App. 325 (2001), 209-220.

[5] P.A. Brunovsky, A classification of linear controllable systems, Kybernetika 3 (1970), 173-187.

[6] F. Bruzelius, S. Petterson, C. Breitholtz, Linear parameter-varying descriptions of non-linear systems, Proc. of the 2004 American Control Conference, pp. 1374-1379.

[7] M.V. Carriegos, R.M. García-Fernández, M.M. López-Cb., M.T. Trobajo, On linear multiinpul systems depending on continuous parameters. Feedback equivalence in low dimension, Int. J. Modern Physics B 26(25) (2012).

[8] M. Carriegos, J.A. Hermida-Alonso and T. SánchezGiralda, The pointwise feedback relation for linear dynamical systems, Linear Algebra Appl. 279 (1998), 119-134.
[9] L. Gillman and M. Jerison, Ring of Continuous Functions, Springer, Berlin, (1976).

[10] J.A. Hermida-Alonso, M.P. Pérez and T. SánchezGiralda, Brunovsky's canonical form for linear dynamical systems over commutative rings, Linear Algebra Appl. 233 (1996), 131-147.

[11] J.A. Hermida-Alonso, M.P. Pérez and T. SánchezGiralda, Feedback invariants for linear Dynamical Systems over a Principal Ideal Domain, Linear Algebra Appl. 218 (1995), 29-45.

[12] J.A. Hermida-Alonso and T. Sánchez-Giralda, Linear equations over commutative rings and determinantal ideals, J. Algebra 99(1) (1986), 72-79.

[13] R.E. Kalman, Kronecker invariants and feedback, in Ordinary Differential Equations. Academic (1972), 459-471.

[14] A.A. Mailybaev, Uncontrollability for linear autonomous multiinput dynamical systems depending on parameters, SIAM J. Control Optimiz. 42(4) (2003) 1431-1450.

[15] Northcott, D.G. Finite Free Resolutions. Cambridge University Press. (1976).

[16] Saez-Schwedt, Andres. (2008), Matricial decomposition of systems over rings, Electronic Journal of Linear Algebra, Volume 17. DOI: http://dx.doi.org/10.13001/1081-3810.1279

[17] E. Sontag, Linear systems over commutative rings: A survey, Ric. di Automatica 7 (1976), 1-34.

[18] E.D. Sontag: Mathematical Control Theory. SpringerVerlag (1990), second edition (1998).

[19] W.A. Wonham, A.S. Morse, Feedback invariants of linear multivariable systems, Automatica 8 (1972), 33100 . 